\title{
Desafios da prática do autocuidado do idoso portador de diabetes mellitus tipo 2
}

\author{
Ernandes Gonçalves Dias \\ Docente na Faculdade Presidente Antônio Carlos de Porteirinha \\ Enfermeiro Especialista em Enfermagem do Trabalho e Docência na Saúde \\ Mestrando do Programa de Inovação e Tecnologia em Enfermagem \\ na Universidade de São Paulo (EERP-USP). \\ $\bowtie$ nandesenf@usp.br \\ Anny Caroliny Silva Pardim \\ Graduanda em Enfermagem \\ Faculdade Presidente Antônio Carlos de Porteirinha \\ Laura Patrícia Antunes \\ Graduanda em Enfermagem \\ Faculdade Presidente Antônio Carlos de Porteirinha \\ Izabela Oliveira Silva \\ Graduanda em Enfermagem \\ Faculdade Presidente Antônio Carlos de Porteirinha \\ Janine Cinara Silveira Alves \\ Docente na Faculdade Presidente Antônio Carlos de Porteirinha \\ Mestre em Ensino de Biologia \\ Sandra Antunes Jorge \\ Enfermeira Especialista em Gestão de Serviços de Saúde com Ênfase em PSF \\ Secretaria Municipal de Saúde de Monte Azul
}

\section{Resumo:}

O Diabetes Mellitus tem se tornado um grande desafio para a saúde pública e está associada à adoção de estilo de vida inadequado, especialmente da má alimentação e o sedentarismo. Objetivou-se identificar os desafios da prática do autocuidado do idoso portador de Diabetes Mellitus tipo 2. Trata-se de um estudo descritivo, com abordagem quantitativa, realizado com 14 idosos de uma Unidade Básica de Saúde do norte do Estado de Minas Gerais. Os dados foram coletados entre março e abril de 2016 por meio de um questionário. Os resultados revelaram que a maioria era do sexo feminino (57,0\%), com idade de 60 a 80 anos $(86,0 \%)$, analfabetos $(50,0 \%)$, pardos $(57 \%)$, casados ou viúvos $(86,0 \%)$, residem com companheiros $(50,0 \%)$, aposentados $(100,0 \%)$ com renda de até 01 salário mínimo (50,0\%). Evidenciou-se que $100,0 \%$ dos idosos realizam tratamento medicamentoso e $79,0 \%$ a monitorização da glicemia como práticas de autocuidado. Os participantes não são tabagistas nem etilistas, porém somente $21,0 \%$ aderem à prática regular de atividade física. Observou-se que, dentre às dificuldades apontadas para a prática do autocuidado, a dificuldade de leitura (71,0\%), alto custo dos medicamentos prescritos pelo médico $(43,0 \%)$ e dificuldade de locomoção até a Unidade de Saúde (43,0\%). Conclui-se ser necessária conscientização dos idosos e profissionais de saúde a respeito dos benefícios da atividade física. A criação de grupos de convivência de idosos para atividades afins e oferta de espaço físico próprio para atividades físicas, podem ser estratégias motivadoras para este público e devem ser planejadas e viabilizadas pelos serviços de saúde. As visitas domiciliares, responsabilização da família com o tratamento do idoso e as ações de educação em saúde podem colaborar positivamente para adoção do autocuidado, e devem ser estratégias de promoção da saúde, considerando as vulnerabilidades do idoso diabético.

Palavras-chave: Diabetes Mellitus; Promoção da Saúde; Autocuidado; Saúde do Idoso. 


\title{
Challenges of the self-care practice of the older carrier of diabetes mellitus type 2
}

\begin{abstract}
:
During the course of the societies' history, the greatest scourges linked to health surveillance resulted from intrinsic factors to the construction of communities, which shows an intimate relationship between health and environmental conditions. The look of biomedicine on human health focus on corrective solutions over preventive ones, because it follows the Cartesian thought with the traditional diagnosis and prophylaxis, conditioned by the commercialization of scientific knowledge. With the purpose of fosteDiabetes Mellitus has become a major public health challenge and is associated with the adoption of inadequate lifestyle, especially poor diet and sedentary lifestyle. The objective of this study was to identify the challenges of the self-care practice of the elderly with type 2 Diabetes Mellitus. This is a descriptive study, with a quantitative approach, carried out with 14 elderly people from a Basic Health Unit in the northern state of Minas Gerais. Data were collected between March and April 2016 by means of a questionnaire. The results showed that the majority were female $(57.0 \%)$, aged $60-80$ years (86.0\%), illiterate (50.0\%), brown (57.0\%), married or widowed (86.0\%), they live with companions (50.0\%), retired (100\%) with income of up to 01 minimum wage (50\%). It was evidenced that $100.0 \%$ of the elderly carried out drug treatment and $79.0 \%$ the monitoring of glycemia as selfcare practices. Participants are not smokers or alcoholics, but only $21.0 \%$ adhere to the regular practice of physical activity. The difficulty of reading (71.0\%), high cost of medications prescribed by the physician (43.0\%) and difficulty in getting to the Health Unit (43.0\%) were observed among the difficulties pointed out for self-care practice. It is concluded that it is necessary to raise awareness among the elderly and health professionals about the benefits of physical activity. The creation of groups for the coexistence of elderly people for related activities and the provision of physical space suitable for physical activities can be motivating strategies for this public and should be planned and made feasible by the health services. Home visits, family responsibility for the treatment of the elderly, and health education actions can positively contribute to the adoption of self-care, and should be strategies for health promotion, considering the vulnerabilities of the elderly diabeticring a critical reflection about the health-disease process, this study defends the need of a paradigm shift concerning biomedicine, defending a break on the hegemony and monopoly of the traditional medicine to mitigate prejudices and allow different therapeutic to the sick.
\end{abstract}

Keywords: Diabetes Mellitus; Health Promotion; Self Care; Health of the Elderly.

\section{Desafíos de la práctica del autocuidado del anciano portador de diabetes mellitus tipo 2}

\begin{abstract}
Resumen:
La diabetes mellitus se ha convertido en un gran desafío para la salud pública y está asociada a la adopción de un estilo de vida inadecuado, especialmente de la mala alimentación y el sedentarismo. Se objetivó identificar los desafíos de la práctica del autocuidado del anciano portador de Diabetes Mellitus tipo 2. Se trata de un estudio descriptivo, con abordaje cuantitativo, realizado con 14 ancianos de una Unidad Básica de Salud del norte del Estado de Minas Gerais. Los datos fueron recolectados entre marzo y abril de 2016 por medio de un cuestionario. Los resultados revelaron que la mayoría era del sexo femenino (57,0\%), con edad de 60 a 80 años $(86,0 \%)$, analfabetos $(50,0 \%)$, pardos $(57,0 \%)$, casados o viudos ( $86 \%)$, residen con compañeros $(50,0 \%)$, jubilados $(100,0 \%)$ con renta de hasta 01 salario mínimo $(50,0 \%)$. Se evidenció que el $100 \%$ de los ancianos realizan tratamiento medicamentoso y el 79,0\% la monitorización de la glucemia como prácticas de autocuidado. Los participantes no son tabaquistas ni etilistas, pero sólo el $21,0 \%$ se adhieren a la práctica regular de actividad física. Se observó que, entre las dificultades apuntadas para la práctica del autocuidado, la dificultad de lectura $(71,0 \%)$, alto costo de los medicamentos prescritos por el médico $(43,0 \%)$ y dificultad de locomoción hasta la Unidad de Salud (43,0\%). Se concluye que es necesaria la concienciación de los ancianos y profesionales de la salud respecto a los beneficios de la actividad física. La creación de grupos de convivencia de ancianos para actividades afines y oferta de espacio físico propio para actividades físicas, pueden ser estrategias motivadoras para este público y deben ser planificadas y viabilizadas por los servicios de salud. Las visitas domiciliarias, la responsabilización de la familia con el tratamiento del anciano y las acciones de educación en salud pueden colaborar positivamente para la adopción del autocuidado, y deben ser estrategias de promoción de la salud, considerando las vulnerabilidades del anciano diabético.
\end{abstract}

Palabras clave: Diabetes Mellitus; Promoción de la Salud; Autocuidado; Salud del Anciano. 


\section{INTRODUÇÃO}

A crescente demanda de pessoas portadoras de Diabetes Mellitus tipo 2 (DM2), representa, atualmente, um importante desafio para os sistemas de saúde de todos os países (FARIA, 2011). Pertence a um grupo de doenças metabólicas, cujo aumento da incidência e prevalência está relacionada com o envelhecimento da população, urbanização crescente e adoção de um estilo de vida inadequado (BRASIL, 2013).

Sabe-se, que há tipos distintos de DM. A classificação proposta pela Organização Mundial da Saúde (OMS) e pela Associação Americana de Diabetes (ADA) inclui quatro classes clínicas: DM1, DM2, DM gestacional e DM associado a outras condições. A DM2 é a forma presente em $90 \%$ a $95 \%$ dos casos e caracteriza-se por defeitos das células beta do pâncreas na qual repercute na ação e secreção da insulina (SOCIEDADE BRASILEIRA DE DIABETES [SBD], 2014; SBD, 2015).

Atualmente, a prevalência de DM2 atinge dimensões epidêmicas, uma vez que esta apresenta uma taxa de morbimortalidade que aumenta gradativamente. O Brasil possui mais de 14 milhões de diabéticos. Dados do Ministério da Saúde apontam que as mortes causadas pelo diabetes estão aumentando, passaram de 24,1 a cada 100 mil habitantes, em 2006, para 28,7, em 2010, ano em que a doença foi relacionada a 54 mil mortes (COSEMS/SP, 2011).

Neste sentido, a população idosa necessita de uma atenção especial, pois, além de apresentarem uma saúde frágil, o acometimento por esta condição prejudica a autonomia, funcionalidade e o ritmo de vida destes (OLIVEIRA, 2010).

Para Nunes, Menezes e Alchieri (2010), no processo de envelhecimento ocorre a instalação de síndromes e fragilidades que tem como principais consequências à imobilidade no leito e a diminuição das capacidades visuais, auditivas, cognitivas e físicas, o que muitas vezes comprometem a autonomia e a independência do idoso.

As doenças decorrentes do processo de envelhecimento geralmente cursam de forma crônica e com crescente dependência, podendo acarretar no indivíduo vários prejuízos como amputações, um número relevante de internações, perdas de mobilidade e perdas irreparáveis na Qualidade de Vida (QV) (QUEIROZ; LEMOS; RAMOS, 2010; BRASIL, 2013). 
O autocuidado pelos portadores de DM é visto como necessário e envolve mudanças comportamentais como nutrição adequada, medicações diárias, exercícios físicos programados, automonitorização da glicemia, cuidados com o próprio corpo e educação permanente, além da perda ou manutenção de peso, não fumar, controle das dislipidemias e da pressão arterial (NAGAI; CHUBACI; NERI, 2012).

Nesse sentido, o autocuidado também é responsabilidade do profissional de saúde e não deve ser entendido como exclusiva responsabilidade do indivíduo e de sua família, mesmo que estes tenham que compreender e aprender a desenvolver atividades de incentivo do autocuidado. Assim, deve ser centrado no indivíduo, utilizando sempre do diálogo para construir conjuntamente o plano de cuidados, levando em consideração as vulnerabilidades da pessoa tendo em vista estabelecer um horizonte comum de cuidados entre todos os atores envolvidos no processo (BRASIL, 2014).

Nesta perspectiva, o paciente idoso portador da DM2, deve ser informado sobre a importância do autocuidado para um tratamento efetivo. Neste sentido, é necessário trabalhar com os idosos portadores dessa condição, orientando-os, afim de que tomem consciência de sua enfermidade e juntamente com sua família e a equipe de saúde, desenvolvam um trabalho com melhor eficiência no controle da hiperglicemia e prevenção dos agravos, adotando desta forma, estilo de vida adequado, quanto ao uso de fármacos corretos, hábitos saudáveis e cuidados com os pés (SBD, 2014).

Diante do exposto julgou-se relevante identificar os desafios da prática do autocuidado do idoso portador de Diabetes Mellitus tipo 2, usuários da Unidade Básica de Saúde Eldorado do município de Porteirinha (MG), uma vez que a adoção deste comportamento pela pessoa idosa pode evitar as complicações relacionadas à sua condição de saúde.

\section{MATERIAL E MÉTODOS}

Trata-se de um estudo descritivo, com abordagem quantitativa, realizado com idosos portadores de DM2 cadastrados na Unidade Básica de Saúde (UBS) Eldorado, localizada na cidade de Porteirinha, norte de Minas Gerais. 
A UBS em estudo é mista, atende o público da zona urbana e rural, porém por questões de logística do estudo foram inclusos no estudo idosos portadores de DM 2 domiciliados na zona urbana da área de abrangência da UBS, no momento da coleta de dados, e exclusos aqueles que não foram encontrados em seu domicílio em até três tentativas durante o período da coleta de dados ou que apresentava alguma limitação cognitiva que o impedia de responder ao questionário. Nesses casos, novo sorteio, manual, de acordo número do prontuário na UBS, foi realizado para compor a amostra.

A população total de idosos portadores de DM2 da UBS era de 62 (zona urbana), considerando os critérios de inclusão, eram elegíveis 46 idosos, porém optou-se trabalhar com $30 \%$ da população, dessa forma a amostra foi constituída por 14 idosos, de ambos os gêneros, independente do estado civil, raça, cor e classe social.

Os idosos participantes foram selecionados de forma aleatória simples e convencional de acordo com a disponibilidade e interesse destes no momento da captação dos dados. Nesse sentido, para compor a amostra foi realizado um sorteio na qual cada idoso foi relacionado pelo nome completo e recebeu um número de um a 47 que na sequência foram recortados e colocados dentro de uma caixa para misturá-los. Prosseguiu-se retirando, sem olhar, um papel de cada vez até que atingiu a quantidade de idosos necessária para o estudo. Antes de iniciar a coleta dos dados, realizou-se, a partir das fichas A, a busca dos idosos diabéticos sorteados para identificar seus respectivos endereços.

Os dados foram coletados no período de março a abril de 2016 na residência do usuário em dia e horário previamente agendado. O questionário foi aplicado pelos pesquisadores na qual os mesmos foram responsáveis pelo seu preenchimento conforme as respostas relatadas pelos entrevistados no momento da coleta.

Para a coleta dos dados, foi utilizado como instrumento um questionário estruturado com questões objetivas que versavam sobre o perfil socioeconômico do idoso portador de DM 2, a prática do autocuidado e as dificuldades encontradas na realização dessa prática.

Para análise dos dados, estes foram digitados, processados e expressos em forma de tabelas utilizando-se uma planilha do Microsoft Excel 2010 e analisados através de Epidemiologia descritiva simples não probabilística. Após análise e interpretação dos dados, foi realizada uma discussão relacionando as informações colhidas com a literatura. 
O estudo obedeceu aos preceitos éticos estabelecidos pela Resolução nº 466, de 12 de dezembro de 2012, do Conselho Nacional de Saúde (CNS), na qual aprova as diretrizes e normas regulamentadoras de pesquisas envolvendo seres humanos. O projeto de pesquisa foi apreciado e aprovado pelo Comitê de Ética em Pesquisa da Associação Educativa do Brasil (CEP SOEBRAS), sob parecer consubstanciado ํำ 1.501.322, Certificado de Apresentação para Apreciação Ética (CAAE) nº 53638616.1.0000.5141. Os idosos participantes do estudo assinaram o Termo de Consentimento Livre e Esclarecido conforme a Resolução supracitada.

\section{RESULTADOS}

Em relação ao gênero, observa-se prevalência feminina (57,0\%). Quanto ao estado civil, houve predominância de sujeitos casados e viúvos (86,0\%). A faixa etária variou entre a idade de 60 a 89 anos, sendo que 86,0\% apresentavam idade entre 60 e 79 anos. Em se tratando do grau de escolaridade, identificou-se que 50,0\% dos entrevistados são analfabetos. Analisando a variável raça/cor, verificou-se que $57,0 \%$ se autodeclararam pardos.

Quando questionados com quem residem, percebeu-se que 50,0\% dos participantes residem com o companheiro. Todos os idosos entrevistados são aposentados e 50,0\% tem renda familiar de até um Salário Mínimo (SM). 
Tabela 1 - Caracterização do perfil socioeconômico e demográfico dos idosos portadores de DM2 cadastrados na UBS Eldorado de Porteirinha (MG), 2016

\begin{tabular}{|c|c|c|}
\hline VARIÁVEIS & $\mathbf{n}$ & $\%$ \\
\hline \multicolumn{3}{|l|}{ Gênero } \\
\hline Feminino & 08 & 57,0 \\
\hline Masculino & 06 & 43,0 \\
\hline \multicolumn{3}{|l|}{ Idade } \\
\hline $60 \vdash 70$ & 06 & 43,0 \\
\hline $70 \vdash 80$ & 06 & 43,0 \\
\hline 80 H 89 & 02 & 14,0 \\
\hline \multicolumn{3}{|l|}{ Escolaridade } \\
\hline Analfabeto & 07 & 50,0 \\
\hline Fundamental incompleto & 06 & 43,0 \\
\hline Médio completo & 01 & 7,0 \\
\hline \multicolumn{3}{|l|}{ Estado Civil } \\
\hline Casado & 06 & 43,0 \\
\hline Viúvo & 06 & 43,0 \\
\hline Divorciado/Desquitado & 02 & 14,0 \\
\hline \multicolumn{3}{|l|}{ Raça/Cor } \\
\hline Pardo & 08 & 57,0 \\
\hline Branco & 05 & 36,0 \\
\hline Negro & 01 & 7,0 \\
\hline \multicolumn{3}{|l|}{ Com quem reside } \\
\hline Companheiro & 07 & 50,0 \\
\hline Outros familiares & 04 & 29,0 \\
\hline Filhos & 02 & 14,0 \\
\hline Sozinho & 01 & 7,0 \\
\hline \multicolumn{3}{|l|}{ Fonte de renda } \\
\hline Aposentadoria & 14 & 100,0 \\
\hline \multicolumn{3}{|l|}{ Renda Familiar } \\
\hline Até $01 \mathrm{SM}$ & 07 & 50,0 \\
\hline 1- $2 \mathrm{SM}$ & 06 & 43,0 \\
\hline $2-3 \mathrm{SM}$ & 01 & 7,0 \\
\hline
\end{tabular}

SM = Salário Mínimo.

Fonte: Dados da pesquisa, 2016. 
Sobre as práticas do autocuidado adotadas pelos idosos portadores de DM2, a Tabela 2 mostra que 100,0\% dos entrevistados fazem uso de medicamentos, 79,0\% monitorizam a glicemia regularmente, $71,0 \%$ realizam consulta médica e de enfermagem, seguem a dieta alimentar voltada para diabético e matem a vacinação em dia, 57,0\% cuidam dos pés.

Tabela 2 - Práticas do autocuidado adotadas pelos portadores de DM2 cadastrados na UBS Eldorado. Porteirinha (MG), 2016

\begin{tabular}{lcccc}
\hline \multirow{2}{*}{ PRÁTICAS DO AUTOCUIDADO } & \multicolumn{2}{c}{ SIM } & \multicolumn{2}{c}{ NÃO } \\
\cline { 2 - 5 } & $\mathbf{n}$ & $\mathbf{0}$ & $\mathbf{n}$ & $\mathbf{\%}$ \\
\hline Faz uso de medicamentos & 14 & 100,0 & - & - \\
Monitorização da glicemia & 11 & 79,0 & 03 & 21,0 \\
Consulta médica e de enfermagem & 10 & 71,0 & 04 & 29,0 \\
$\begin{array}{l}\text { Segue dieta alimentar voltada } \\
\text { para DM }\end{array}$ & 10 & 71,0 & 04 & 29,0 \\
Mantém vacinação em dia & 10 & 71,0 & 04 & 29,0 \\
Cuidado com os pés & 08 & 57,0 & 06 & 43,0 \\
Pratica exercício físico regular & 03 & 21,0 & 11 & 79,0 \\
Participa de grupos operativos & 03 & 21,0 & 11 & 79,0 \\
\hline
\end{tabular}

Fonte: Dados da pesquisa, 2016.

No que diz respeito aos hábitos de vida dos idosos portadores de DM2, a Tabela 3 mostra não possuírem o hábito de fumar ou consumir bebidas alcoólicas, o que é bastante relevante para um tratamento mais eficaz, porém 79,0\% não praticam atividade física.

Tabela 3 - Hábitos de vida dos portadores de DM2 cadastrados na UBS Eldorado. Porteirinha (MG), 2016

\begin{tabular}{|c|c|c|c|c|}
\hline \multirow{2}{*}{ HÁBITOS DE VIDA } & \multicolumn{2}{|c|}{ SIM } & \multicolumn{2}{|c|}{ NÃO } \\
\hline & $\mathbf{n}$ & $\%$ & $\mathbf{n}$ & $\%$ \\
\hline Tabagista & - & - & 14 & 100,0 \\
\hline Etilista & - & - & 14 & 100,0 \\
\hline Atividade física & 03 & 21,0 & 11 & 79,0 \\
\hline
\end{tabular}

Fonte: Dados da pesquisa, 2016. 
Quando questionados sobre as dificuldades encontradas para a prática do autocuidado, observa-se na Tabela 4 que 71,0\% dos idosos têm dificuldade de leitura, 43,0\% tem como dificuldade o alto custo dos medicamentos prescritos pelo médico e dificuldade de locomoção até a UBS. A baixa renda e enfermidades associadas à DM2 é uma dificuldade que interfere na adoção de hábitos para 28,0\% dos participantes, 21,0\% relacionam as dificuldades de praticar o autocuidado à idade avançada, desconhecimento sobre a doença, aos efeitos colaterais do tratamento medicamentosos e a falta de acesso aos medicamentos gratuitos.

Tabela 4 - Dificuldades encontradas na prática do autocuidado, pelos idosos portadores de DM2 cadastrados na UBS Eldorado de Porteirinha (MG), 2016

\begin{tabular}{|c|c|c|c|c|}
\hline \multirow{2}{*}{$\begin{array}{c}\text { DIFICULDADES NA PRÁTICA DO } \\
\text { AUTOCUIDADO }\end{array}$} & \multicolumn{2}{|c|}{ SIM } & \multicolumn{2}{|c|}{ NÃO } \\
\hline & $\mathbf{n}$ & $\%$ & $\mathbf{n}$ & $\%$ \\
\hline Dificuldade de leitura & 10 & 71,0 & 04 & 29,0 \\
\hline $\begin{array}{l}\text { Custo alto dos medicamentos prescritos } \\
\text { pelo médico }\end{array}$ & 06 & 43,0 & 08 & 57,0 \\
\hline Dificuldade de locomoção até a UBS & 06 & 43,0 & 08 & 57,0 \\
\hline Baixa renda & 04 & 28,0 & 10 & 72,0 \\
\hline Enfermidades associadas & 04 & 28,0 & 10 & 72,0 \\
\hline Idade avançada & 03 & 21,0 & 11 & 79,0 \\
\hline Falta de conhecimento sobre a doença & 03 & 21,0 & 11 & 79,0 \\
\hline Efeitos colaterais dos medicamentos & 03 & 21,0 & 11 & 79,0 \\
\hline $\begin{array}{l}\text { Falta de acesso aos medicamentos } \\
\text { gratuitos }\end{array}$ & 03 & 21,0 & 11 & 79,0 \\
\hline $\begin{array}{l}\text { Relacionamento inadequado com os } \\
\text { profissionais de saúde }\end{array}$ & 02 & 14,0 & 12 & 86,0 \\
\hline $\begin{array}{l}\text { Tempo de espera prolongado para } \\
\text { atendimento na UBS }\end{array}$ & 02 & 14,0 & 12 & 86,0 \\
\hline $\begin{array}{l}\text { Dificuldade em entender a linguagem } \\
\text { dos profissionais de saúde }\end{array}$ & 01 & 7,0 & 13 & 93,0 \\
\hline Falta de apoio familiar & 01 & 7,0 & 13 & 93,0 \\
\hline $\begin{array}{l}\text { Preguiça para realizar atividade física } \\
\text { regularmente }\end{array}$ & 01 & 7,0 & 13 & 93,0 \\
\hline
\end{tabular}

Fonte: Dados da pesquisa, 2016. 


\section{DISCUSSÃO}

Observou-se prevalência de idosos portadores de DM2 do sexo feminino quando comparado ao montante de idosos pesquisados. Essa ascendência vem corroborar, com os dados encontrados por Barbosa, Matos e Sousa (2013), em uma pesquisa realizada com idosos diabéticos usuários de uma Estratégia Saúde da Família de Teresina-PI para avaliar o autocuidado, foi constatado que 58,2\% dos entrevistados era representada por mulheres.

A predominância do sexo feminino no grupo de idosos portadores de DM2 é também confirmada por um estudo realizado por Lucena et al. (2014), em uma amostra de 22 idosos portadores de DM2 participantes do projeto de pesquisa e extensão "Envelhecimento saudável", desenvolvido na Faculdade de Enfermagem e Medicina Nova Esperança, na cidade de João Pessoa (PB), no ano de 2013, na qual verificaram que 90,0\% dos entrevistados são do sexo feminino.

Em relação à idade, os dados corroboram com os encontrados por Sampaio (2012), ao realizar uma pesquisa com portadores de DM2 atendidos em um serviço especializado para referência no atendimento a pacientes com DM de Fortaleza (CE), observou que 40,4\% dos participantes tinham de 60 a 70 anos, $23,3 \%$ de 70 a 80 anos e $8,8 \%$ com idade igual ou superior a 80 anos.

A incidência de DM2 aumenta proporcionalmente com o advento da idade, assim com o atual cenário mundial de envelhecimento populacional e aumento da expectativa de vida, estima-se o crescimento na quantidade de indivíduos acometidos por esta condição crônica de saúde para as próximas décadas (BOSI et al., 2009).

Percebeu-se que a maioria dos idosos portadores de DM2 tem um baixo nível de escolaridade, o que pode dificultar a sua adesão ao tratamento e o entendimento em relação à doença e aos cuidados. Esses dados são similares aos encontrados por Carolino et al. (2008) em um estudo realizado com 66 idosos portadores de DM2 cadastrados numa UBS na cidade de Maringá (PR) em que verificaram que a maioria dos entrevistados apresentaram baixo nível de escolaridade, sendo que 37,88\% não eram alfabetizados, 30,3\% estudaram até a $4^{\text {a }}$ série, $16,67 \%$ concluíram o ensino fundamental e 12,12 e 3,03\% cursaram o ensino médio e o ensino superior, respectivamente. 
Ainda em relação à escolaridade, os dados desse estudo apresentam-se discordantes quando comparados aos resultados de Cruz, Melo e Barbosa (2011), onde foi evidenciado que 49,9\% dos idosos entrevistados, naquele estudo, tinham acima de oito anos de estudos (fundamental completo), refletindo um grau de escolaridade satisfatório, o que proporciona o melhor controle do DM2, aumentando a adesão ao tratamento e, diminuindo os riscos de complicações decorrentes da doença.

Os entrevistados eram casados, viúvos ou separados. Nesse sentido, esses dados assemelham-se aos encontrados por Goulart (2013) em um estudo com 50 pessoas portadoras de DM2 cadastradas numa ESF em Itajubá-MG, em que 46,0\% dos participantes eram casados, $36,0 \%$ viúvos e $18,0 \%$ separados/solteiros.

Em relação à cor, em uma pesquisa similar realizada com 26 pacientes portadores de DM2 em um centro de saúde do Distrito de Saúde Norte em Campinas, os pesquisadores Melo e Campos (2014) obtiveram que 46,2\% dos entrevistados se autorreferiram pardos e $38,5 \%$, brancos.

$\mathrm{Na}$ avaliação da variável "com quem o idoso reside", o resultado é discordante dos encontrados por Santos et al. (2013), em um estudo realizado no município de Uberaba (MG) com idosos portadores de DM, onde encontraram que 22,3\% dos participantes moravam com o cônjuge, $34,6 \%$ com os filhos e $10,0 \%$ moram sozinhos.

Quanto à renda, os dados deste estudo são discordantes dos encontrados por Sampaio (2012) em sua pesquisa com portadores de DM2 atendidos em num serviço especializado para referência no atendimento a pacientes com DM de Fortaleza (CE) na qual $66,8 \%$ eram aposentados, 27,2\% ainda trabalhavam, 5,2\% estavam desempregados e os demais exerciam atividades do lar.

A maioria $(50,0 \%)$ dos idosos entrevistados afirmou ter renda familiar até um SM. Esses dados são semelhantes aos encontrados por Marques et al. (2013), em seu estudo realizado com 100 idosos com diagnóstico de DM2 em Centros de Saúde da Família em Fortaleza (CE) no qual observou que 36,0\% dos portadores de DM 2 recebiam de um a dois SM.

Sobre a prática de autocuidado, os dados apontam que todos os idosos portadores de DM2 entrevistados adere ao tratamento medicamentoso. Esse resultado é concordante aos 
encontrados no estudo realizado por Faria et al. (2013) com usuários portadores de DM na região sudeste do Brasil, em que encontrou entre os sujeitos investigados que $84,4 \%$ apresentaram adesão ao tratamento medicamentoso para DM2.

Conforme Farias (2011), os portadores de DM, comumente, necessitam de terapêutica medicamentosa, pois geralmente apresentam dificuldades em conseguir o controle da doença apenas com as medidas não medicamentosas. Frente às complicações da doença, é recomendável que ambos os tratamentos sejam realizados concomitantemente.

A prática medicamentosa é essencial para controle do DM e pressupõe o conhecimento acerca da medicação prescrita para que venha otimizar o tratamento. Dessa forma, os profissionais devem orientar os pacientes quanto à terapêutica medicamentosa, sua eficácia, o nome comercial do medicamento, dose e horário de administração, seu mecanismo de ação e possíveis efeitos colaterais (VISENTIN et al., 2016).

Porém, o controle da glicemia está diretamente relacionado a uma alimentação adequada na qual se deve englobar o cumprimento de horários e ingestão de porções em quantidades corretas. Para tanto, deve-se elaborar um plano alimentar individual de acordo com a realidade de cada paciente, de forma a facilitar o seu interesse em realizá-lo e fornecer todas as informações necessárias para facilitar o seu entendimento (LIMA et al., 2013).

Observou-se que, em relação aos hábitos de vida, há predominância de indivíduos que têm hábitos saudáveis, uma vez, que não são fumantes e não consomem bebidas alcoólicas, porém a maioria não são adeptos à prática de exercício físico. Resultados concordantes foram encontrados por Pereira, Cardoso e Moraes (2011) em um estudo com indivíduos portadores de DM2 atendidos numa UBS de Várzea Grande-MT que também confirmaram ter hábitos saudáveis, visto que $80,8 \%$ dos participantes afirmaram não fumar, $76,9 \%$ relataram não consumir nenhum tipo de bebida alcoólica e 84,6\% não praticam nenhuma atividade física.

Segundo Brasil (2013), mudanças no de estilo de vida através da adoção de hábitos de vida saudáveis, tais como: uma alimentação equilibrada, prática regular de atividade física, moderação no uso de álcool e abandono do tabagismo são a base do tratamento do DM2, além de possuir uma importância fundamental no controle glicêmico e na prevenção das complicações crônicas das doenças. 
Em relação à dificuldade para realização do autocuidado, Chagas et al. (2013) em seu estudo com portadores DM2, usuários da UBS do Distrito Sanitário Leste de Belo Horizonte $(\mathrm{MG})$, identificou que os entrevistados têm como barreiras a falta de disciplina e falta de apoio da família, restrição de alimentos, estresse, trabalho, segurança e geografia do local de moradia que inviabiliza a atividade física, dores articulares e doenças não associadas a doença; além de relatarem a dificuldade da mudança de hábitos de vida em que estavam acostumados até descobrirem o diagnóstico da doença. A questão financeira também foi apontada como empecilho para o autocuidado, por acreditarem dificultar o acesso aos alimentos recomendados na dieta.

Silva et al. (2013), em uma pesquisa realizada com 27 usuários diabéticos cadastrados numa ESF de Ilhéus (BA), encontraram que o principal fator dificultador ao processo de tratamento e autocuidado é a negligência do próprio paciente, o qual abandona as orientações do profissional de saúde. Além disso, a falta de condições financeiras e reações adversas aos fármacos também foram mencionadas como dificultadores.

\section{CONCLUSÃO}

Evidenciou-se que os idosos adotam o tratamento medicamentoso e não medicamentoso como elementos integrantes das práticas de autocuidado. 0 estilo de vida adotado parece coerente com as recomendações das políticas públicas do Ministério da Saúde, com boas práticas de autocuidado, contudo a atividade física ainda precisa ser incentivada pelos profissionais de saúde.

Nesse sentido, sugere-se a conscientização dos idosos e profissionais de saúde a respeito dos benefícios da atividade física para diabéticos. A criação de grupos de convivência de idosos para atividades afins e oferta de espaço físico próprio para atividades físicas, podem ser estratégias motivadoras para este público e devem ser planejadas e viabilizadas pelos serviços de saúde.

Alguns fatores pessoais, familiares, sociais, econômicos, demográficos e de acesso ao serviço de saúde parecem dificultarem, em algum momento, a prática do autocuidado, por isso precisam ser considerados na elaboração de políticas públicas de saúde e 
oportunamente em toda abordagem do idoso na Unidade de Saúde ou no cenário da comunidade.

As visitas domiciliares, responsabilização da família com o tratamento do idoso, as ações de educação em saúde podem colaborar positivamente para adoção do autocuidado, assim, considerando a importância da adesão dos idosos portadores de DM2 às práticas de autocuidado e as dificuldades apontadas, julga-se ser necessária concentração de esforços por parte dos profissionais da saúde e gestores no planejamento e elaboração de estratégias de intervenções que considerem as vulnerabilidades do idoso diabético.

Espera-se que esse estudo possa auxiliar o profissional de saúde no planejamento de intervenções educativas para que os idosos compreendam a importância do autocuidado e sintam-se motivados a realizá-lo.

\section{REFERÊNCIAS}

BARBOSA, C. de O.; MATOS, R. R.; SOUSA, R. W. F. de M. Avaliação do autocuidado em portadores de diabetes na maturidade de uma Estratégia Saúde da Família de Teresina-PI. ConScientiae Saúde, Teresina, v. 12, n. 1, p. 128-136, dez. 2013.

BOSI, P. L.; CARVALHO, A. M.; CONTRERA, D.; CASALE, G.; PEREIRA, M. A.; GRONNER, M. F.; DiOGO, T. M.; TORQUARTO, M. T. da C. G.; OISHI, J.; LEAL, A. M. de O. Prevalência de diabetes melito e tolerância à glicose diminuída na população urbana de 30 a 79 anos da cidade de São Carlos, São Paulo. Arq Bras Endocrinol Metab, São Paulo, v. 53, n. 6, p. 726-732, ago. 2009.

BRASIL, Ministério da Saúde. Secretaria de Atenção à Saúde. Departamento de Atenção Básica. Estratégias para o cuidado da pessoa com doença crônica Diabetes Mellitus. Brasília: Ministério da Saúde, 2013. 162p. (Cadernos de Atenção Básica, n. 36).

BRASIL. Ministério da Saúde. Secretaria de Atenção à Saúde. Departamento de Atenção Básica. Estratégias para o cuidado da pessoa com doença crônica. Brasília: Ministério da Saúde, 2014. 162 p. (Cadernos de Atenção Básica, n. 35).

CAROLINO, I. D. R.; MOLENA-FERNADES, C. A.; TASCA, R. S.; MARCON, S. S.; CUMAN, R. K. N. Fatores de risco em pacientes com diabetes mellitus tipo 2. Rev. Latino-Am. Enfermagem, Ribeirão Preto , v. 16, n. 2, p. 238-244, abr. 2008 .

CHAGAS, C. A.; TORRES, H. de C.; MELO, S. de M.; PEREIRA, P. de F.; SIMAN, J. B. As barreiras das práticas de autocuidado: desafios e oportunidades para o empoderamento em grupos de diabetes na atenção primária. In: II Congresso Online - Gestão, Educação e Promoção da Saúde, 2013. II Congresso Online - Gestão, Educação e Promoção da Saúde, 2013.

COSEMS/SP. Conselho de Secretários Municipais de Saúde do Estado de São Paulo. Em defesa do SUS. Audiência Pública: A realidade do tratamento do diabetes no Brasil e sua judicialização, 2011. [internet]. 
Disponível em: <http://www.cosemssp.org.br/noticias/591/audiencia-publica-a-realidade-do-tratamento-dodiabetes-no-brasil-e-sua-judicializacao.html>. Acesso em: 05 jun. 2017.

CRUZ, A. R. R.; MELO, C. M. A.; BARBOSA, C. O. Conhecimentos dos diabéticos sobre a doença e o tratamento nutricional. RBCEH, Passo Fundo, v. 8, n. 3, p. 343-354, set./dez. 2011.

FARIA, H. T. G.; RODRIGUES, F. F. L.; ZANETTI, M. L.; ARAÚJO, M. F. M. de; DAMASCENO, M. M. C. Fatores associados à adesão ao tratamento de pacientes com diabetes mellitus. Acta Paul Enferm. v 26, n 3, p. 231-237, jun. 2013.

FARIAS, H. T. G. Desafios para atenção em saúde: adesão ao tratamento e controle metabólico em pessoas com diabetes melitus tipo 2, no município de Passos -MG. 2011. 170p. Tese doutorado (Escola de Enfermagem de Ribeirão Preto) Universidades de São Paulo. Preto. Ribeirão Preto. 2011.

GOULART, J. C. M. Avaliação das ações de autocuidado da pessoa portadora de diabetes mellitus. 2013. 80f. Monografia (Graduação em Enfermagem). Escola de Enfermagem Wenceslau Brás, Itajubá - MG, 2013.

LIMA, A. A.; SANTANA, A. V. de; AZEVEDO, B. F. de; CORREIA, N. R.; ROCHA, R. C. de O. R.; CORREIA, M. das G. da S. A importância da dieta hospitalar na recuperação de pacientes diabéticos. Cadernos de Graduação Ciências Biológicas e da Saúde, v. 1, n. 16, p. 47-56, 2013.

LUCENA, A. L. R.; FREITAS, F. F. Q.; LUCENA, A. V. de F.; MACEDO, P. D. de; MEDEIROS, R. B. de; FERREIRA, M. das G. N. O autocuidado dos idosos e a redução das complicações da Diabetes Melittus tipo 2. Rev. Ciênc. Saúde Nova Esperança, Nova Esperança, v.12, n. 2, p. 1-10, dez. 2014.

MARQUES, M. B.; SILVA, M. J. da S.; COUTINHO, J. F. V.; LOPES, M. V. de O. Avaliação da competência de idosos diabéticos para o autocuidado. Rev. esc. enferm. USP, São Paulo, v. 47, n. 2, p. 415-420, abr. 2013.

MELO, L. P. de ; CAMPOS, E. A. de . "O grupo facilita tudo": significados atribuídos por pacientes portadores de diabetes mellitus tipo 2 a grupos de educação em saúde . Revista Latino-Americana de Enfermagem, São Paulo, v. 22, n. 6, p. 980-987, dez. 2014.

NAGAI, P. A.; CHUBACI, R. Y. S.; NERI, A. L. Idosos diabéticos: as motivações para o autocuidado. Revista Temática Kairós Gerontologia,São Paulo, v. 15, n. 6, p. 407-434, Dez. 2012.

NUNES, V. M. A; MENEZES, R. M. P.; ALCHIERI, J. C. Avaliação da Qualidade de Vida em Idosos Institucionalizados no Município de Natal, Estado do Rio Grande do Norte. Acta Scientiarum. Health Sciences, Maringá, v. 32, n. 2, p. 119-126, 2010.

OLIVEIRA, J. P. Portador de Diabetes Mellitus Tipo 2: mudança de hábitos para adesão ao tratamento. 2010. 65p. Monografia (Graduação em Enfermagem). Faculdade Tecsoma. Paracatu. 2010.

PEREIRA, J. G.; CARDOSO, M. I.; MORAES, M. A. M. Práticas de autocuidado desenvolvidas por diabéticos tipo 2 em uma Unidade Básica de Saúde da Família. Gestão \& Saúde, Brasilia, DF. Brasil, v. 2, n. 1, p. 277-290, out. 2011.

QUEIROZ, Z. P. V.; LEMOS, N. F. D.; RAMOS, L. R. Fatores potencialmente associados à negligência doméstica entre idosos atendidos em programa de assistência domiciliar. Ciência \& Saúde Coletiva, v. 15, n. 6, p. 2815$24,2010$. 
SAMPAIO, C. F. S. Práticas de autocuidado de pessoas com diabetes mellitus tipo 2: implicações para o cuidado clínico e educativo de enfermagem. 2012. 134p. Dissertação (Mestrado Acadêmico em Cuidados Clínicos em Enfermagem e Saúde). Universidade Estadual do Ceará, Fortaleza-CE, 2012..

SANTOS, É. A. dos; TAVARES, D. M. dos S.; RODRIGUES, L. R.; DIAS, F. A.; FERREIRA, P. C. dos S. Morbidades e qualidade de vida de idosos com diabetes mellitus residentes nas zonas rural e urbana. Rev. esc. enferm. USP [online], v. 47, n. 2, p.393-400, 2013. Disponível em: http://www.scielo.br/scielo.php? script=sci arttext\&pid=S0080-62342013000200017. Acesso em: mai. 2016.

SILVA, B. C.; BRAZ, C. A.; ROTONDANO, J. A. R.; SILVA, M. P.; CALDAS, N. M. Avaliação da adesão ao tratamento pelo paciente com Diabetes Mellitus na Estratégia de Saúde da Família Basílio I do município de Ilhéus, Bahia, Brasil. UDESC em Ação. Ihéus, v. 7, n. 1, 2013.

SOCIEDADE BRASILEIRA DE DIABETES (SBD). Diretrizes da sociedade brasileira de diabetes 2013-2014. 3 ed. São Paulo: A. C Farmacêutica, 2014. 365p. Disponível em: https://www.diabetes.org.br/images/pdf/diretrizessbd.pdf. Acesso em: out. 2016.

SOCIEDADE BRASILEIRA DE DIABETES (SBD). Diretrizes da sociedade brasileira de diabetes (2014-2015), São Paulo. AC Farmacêutica. 2015. Disponível em: https://www.diabetes.org.br/images/2015/arearestrita/diretrizes-sbd-2015.pdf. Acesso em: jul. 2016.

VISENTIN, A.; MANTOVANI, M. de F.; CAVEIÃO, C.; HEY, A. P.; SCHENEIDER, E. P.; PAULINHO, V. Autocuidado de usuários com diabetes tipo 1 em uma unidade básica de saúde. Rev enferm UFPE on line., Recife, v 10, n 3, p.991-998, mar. 2016. Disponível em: http://www.revista.ufpe.br/revistaenfermagem/index.php/revista/index. Acesso em: ago. 2016. 